PROCEEDINGS OF THE

AMERICAN MATHEMATICAL SOCIETY

Volume 130, Number 10, Pages 3017-3023

S 0002-9939(02)06554-1

Article electronically published on May 8, 2002

\title{
HELGASON-MARCHAUD INVERSION FORMULAS FOR RADON TRANSFORMS
}

\author{
BORIS RUBIN
}

(Communicated by David Preiss)

\begin{abstract}
Let $X$ be either the hyperbolic space $\mathbb{H}^{n}$ or the unit sphere $S^{n}$, and let $\Xi$ be the set of all $k$-dimensional totally geodesic submanifolds of $X, 1 \leq k \leq n-1$. For $x \in X$ and $\xi \in \Xi$, the totally geodesic Radon transform $f(x) \rightarrow \hat{f}(\xi)$ is studied. By averaging $\hat{f}(\xi)$ over all $\xi$ at a distance $\theta$ from $x$, and applying Riemann-Liouville fractional differentiation in $\theta$, S. Helgason has recovered $f(x)$. We show that in the hyperbolic case this method blows up if $f$ does not decrease sufficiently fast. The situation can be saved if one employs Marchaud's fractional derivatives instead of the Riemann-Liouville ones. New inversion formulas for $\hat{f}(\xi), f \in L^{p}(X)$, are obtained.
\end{abstract}

\section{INTRODUCTION}

Following [6, p. 96], for $\theta>0$ we denote

$$
\hat{f}_{\theta}(\xi)=\int_{d(x, \xi)=\theta} f(x) d m(x), \quad \check{\varphi}_{\theta}(x)=\int_{d(x, \xi)=\theta} \varphi(\xi) d \mu(\xi),
$$

where $d(\cdot, \cdot)$ designates a geodesic distance, and $d \mu(x)$ and $d m(\xi)$ are the relevant normalized measures. For $\theta=0$ operators (1.1) coincide with the Radon transform $\hat{f}(\xi)$ and its dual $\check{\varphi}(x)$. Helgason ([5], [6, p. 97]) has proved the following formulae:

$$
\begin{aligned}
& f(x)=c\left[\left(\frac{d}{d\left(u^{2}\right)}\right)^{k} \int_{0}^{u}(\hat{f})_{\cosh ^{-1}\left(v^{-1}\right)}^{\vee}(x)\left(u^{2}-v^{2}\right)^{k / 2-1} d v\right]_{u=1}, \quad X=\mathbb{H}^{n}, \\
& f(x)=\frac{c}{2}\left[\left(\frac{d}{d\left(u^{2}\right)}\right)^{k} \int_{0}^{u}(\hat{f})_{\cos ^{-1}(v)}^{\vee}(x) v^{k}\left(u^{2}-v^{2}\right)^{k / 2-1} d v\right]_{u=1}, \quad X=S^{n} .
\end{aligned}
$$

Here $f \in C_{c}^{\infty}\left(\mathbb{H}^{n}\right)$ or $f \in C^{\infty}\left(S^{n}\right), c^{-1}=(k-1) ! \sigma_{k} / 2^{k+1}, \sigma_{k}$ is the area of the unit sphere $S^{k} \subset \mathbb{R}^{k+1}$.

Question. Are (1.2) and (1.3) applicable to $f \in L^{p}(X)$ ?

For (1.3) the answer is "yes" 9] provided that all derivatives are understood in the $L^{p}$-norm or in the a.e. sense. In the noncompact case (1.2) the answer is in

Received by the editors May 16, 2001.

2000 Mathematics Subject Classification. Primary 44A12; Secondary 52A22.

Key words and phrases. Geodesic Radon transforms, Marchaud's fractional derivatives.

This work was partially supported by the Edmund Landau Center for Research in Mathematical Analysis and Related Areas, sponsored by the Minerva Foundation (Germany).

(C)2002 American Mathematical Society 
general "no" by the following reason. It is known [3] that for $f \in L^{p}\left(\mathbb{H}^{n}\right), \hat{f}(\xi)$ exists for almost all $\xi \in \Xi$ if and only if

$$
1 \leq p<(n-1) /(k-1) .
$$

The point is that the integral in (1.2) can be divergent even if $\hat{f}$ is well defined.

In the present article we suggest "regularized versions" of (1.2) which work well for all $f \in L^{p}\left(\mathbb{H}^{n}\right)$ where $p$ obeys (1.4). Our method relies on implementation of Marchaud's fractional derivatives. In particular, for $k=1$ the following simple inversion formulae hold.

Theorem A. Let $f \in L^{p}\left(\mathbb{H}^{n}\right), 1 \leq p<\infty$. Then

$$
f=\frac{1}{\pi} \int_{0}^{\infty} \frac{(\hat{f})^{\vee}-(\hat{f})_{\theta}^{\vee}}{\sinh ^{2} \theta} \cosh \theta d \theta, \quad \int_{0}^{\infty}=\lim _{\varepsilon \rightarrow 0} \int_{\varepsilon}^{\infty}
$$

where the limit is understood in the $L^{p}$-norm and in the a.e. sense. If $f \in C_{0}\left(\mathbb{H}^{n}\right) \cap$ $L^{p}\left(\mathbb{H}^{n}\right)$, where $C_{0}\left(\mathbb{H}^{n}\right)$ is the space of continuous functions vanishing at infinity, then $\lim _{\varepsilon \rightarrow 0}$ can be treated in the sup-norm.

Theorem B. Let $f \in L^{p}\left(S^{n}\right), 1 \leq p<\infty$. Then

$$
f=\frac{(\hat{f})^{\vee}}{2 \pi}+\frac{1}{2 \pi} \int_{0}^{\pi / 2} \frac{(\hat{f})^{\vee}-(\hat{f})_{\theta}^{\vee}}{\sin ^{2} \theta} \cos \theta d \theta, \quad \int_{0}^{\pi / 2}=\lim _{\varepsilon \rightarrow 0} \int_{\varepsilon}^{\pi / 2}
$$

where the limit is understood in the $L^{p}$-norm and in the a.e. sense. If $f \in C\left(S^{n}\right)$, then $\lim _{\varepsilon \rightarrow 0}$ is uniform.

Analogous formulae for $k>1$ envolve finite differences of higher orders and/or derivatives which must be understood in a certain $L^{q}$-norm or in the a.e. sense (see Theorems 2.2, 2.4, and 3.3 below, and also [9, Section 4.2]). These formulae are inevitably more cumbersome in accordance with the general theory of fractional integrals [8].

Historical remark. Apart from (1.2) and (1.3), Helgason 4] suggested another inversion formula $f=P_{k / 2}(\Delta)(\hat{f})^{\vee}$, in which $k$ is even, $f$ is smooth, and $P_{k / 2}(\Delta)$ is a polynomial of the Laplace-Beltrami operator $\Delta$. This formula was extended to all $k$ by Berenstein and Casadio Tarabusi (for $X=\mathbb{H}^{n}$ ) in [1] and by the author (for $X=S^{n}, \mathbb{H}^{n}$ ) in 9, 10, using different approaches. Investigation of Radon transforms on $L^{p}$-spaces was initiated by Oberlin and Stein [7], and Strichartz [12. For $f \in L^{p}, \hat{f}$ can be inverted explicitly by making use of continuous wavelet transforms and the convolution-backprojection method [2, 3] 9, 11]. Applicability of (1.2) to $f \in L^{p}$ was not studied before. We complete this gap below. References to the relevant important works by S. Gindikin, E. Grinberg, S. Ishikawa, T. Kakehi, A. Kurusa, E.T. Quinto and others can be found in [2, 3, 6, 9, 10, 11.

\section{InVersion formulae of the Marchaud type}

2.1. The case $X=\mathbb{H}^{n}$. We start with the following equality:

$$
\sigma_{k-1} \int_{0}^{\infty} F(\cosh \theta \cosh r) \sinh ^{k-1} r d r=\hat{F}(\cosh \theta),
$$


which is due to Helgason [5, 6] in the smooth set-up. Here

$$
\begin{aligned}
& F(t)=\left(M_{t} f\right)(x)=\frac{\left(t^{2}-1\right)^{(1-n) / 2}}{\sigma_{n-1}} \int_{\{y \in X:[x, y]=t\}} f(y) d \sigma(y), \\
& {[x, y]=-x_{1} y_{1}-\ldots-x_{n} y_{n}+x_{n+1} y_{n+1} ; \quad \hat{F}(\cosh \theta)=(\hat{f})_{\theta}^{\vee}(x) .}
\end{aligned}
$$

From this point our argument becomes different. The first question is whether (2.1) is true for generic $f \in L^{p}$. By setting $u=\cosh \theta, v=\cosh \theta \cosh r$, we write (2.1) as

$$
\sigma_{k-1} \int_{u}^{\infty} F(v)\left(v^{2}-u^{2}\right)^{k / 2-1} d v=u^{k-1} \hat{F}(u) .
$$

The left-hand side of (2.3) represents a hyperbolic convolution of the form

$$
(k * f)(x)=\int_{X} f(y) k([x, y]) d y
$$

with $k(\tau)=\sigma_{k-1} \sigma_{n-1}^{-1}\left(\tau^{2}-u^{2}\right)_{+}^{k / 2-1}\left(\tau^{2}-1\right)^{1-n / 2}$ (we use a standard notation $a_{+}^{\lambda}=a^{\lambda}$ if $a>0$ and 0 otherwise). By Young's inequality

$$
\begin{gathered}
\|k * f\|_{q} \leq\|f\|_{p}\|k\|_{r}, \quad 1 \leq p \leq q \leq \infty, \quad 1-\frac{1}{p}+\frac{1}{q}=\frac{1}{r}, \\
\|k\|_{r}^{r}=\sigma_{n-1} \int_{1}^{\infty}|k(\tau)|^{r}\left(\tau^{2}-1\right)^{n / 2-1} d \tau .
\end{gathered}
$$

The left-hand side of (2.3) (written as (2.4)) is a linear bounded operator from $L^{p}$ to $L^{q}$ provided $0 \leq q^{-1}<p^{-1}-(k-1) /(n-1)$. This condition agrees with (1.4). Thus for all $f \in L^{p}$ with $p$ in the maximal range $1 \leq p<(n-1) /(k-1)$, the left-hand side of (2.3) is finite for all $u>0$ and almost all $x$. This justifies all operations having been used in [5, 6] for derivation of (2.1).

In order to recover $f$ we write $(2.3)$ in the form

$$
\begin{gathered}
\left(I_{-}^{k / 2} g_{x}\right)(u)=\psi_{x}(u), \\
g_{x}(v)=v^{-1 / 2}\left(M_{\sqrt{v}} f\right)(x), \quad \psi_{x}(u)=\frac{u^{(k-1) / 2}}{\pi^{k / 2}} \check{\varphi}_{\cosh ^{-1} \sqrt{u}}(x),
\end{gathered}
$$

where $\left(I_{-}^{\alpha} g\right)(u)=\frac{1}{\Gamma(\alpha)} \int_{u}^{\infty} g(v)(v-u)^{\alpha-1} d v$ denotes the Weyl fractional integral of order $\alpha$. A simple calculation yields

$$
\left(I_{-}^{\alpha} g_{x}\right)(u)=\frac{2}{\Gamma(\alpha) \sigma_{n-1}} \int_{X} f(y)\left([x, y]^{2}-u\right)_{+}^{\alpha-1}\left([x, y]^{2}-1\right)^{1-n / 2} d y,
$$

and by $(2.5)$, for $1 \leq p \leq \infty$,

$$
\left\|I_{-}^{\alpha} g_{x}\right\|_{q_{\alpha}} \leq c\|f\|_{p}, \quad 0 \leq \frac{1}{q_{\alpha}}<\frac{1}{p}-\frac{2 \alpha-1}{n-1} .
$$

The condition

$$
(2 \alpha-1) /(n-1)<1 / p \leq 1
$$

is necessary for the existence of (2.8). It means that implementation of the semigroup property $I_{-}^{\alpha} I_{-}^{\beta}=I_{-}^{\alpha+\beta}$ (as in [5, 6]) may lead to divergent integrals. 
Example 2.1. For $k=1$ the Radon transform $\hat{f}, f \in L^{p}$, is well defined for all $p \in[1, \infty)$. Formally $I_{-}^{1 / 2} I_{-}^{1 / 2} g_{x}=I_{-}^{1} g_{x}$. By (2.10) with $\alpha=1$ the integral $I_{-}^{1} g_{x}$ can be divergent if $p \geq n-1$. The case $n=2$ when divergence occurs for all $p \geq 1$ is especially dramatic.

In order to avoid unnatural restrictions on $k, p$ and $n$, we invert (2.6) with the aid of Marchaud's fractional derivative as follows (cf. [8, p. 157]). Let $\alpha=k / 2$. Given a positive integer $\ell>\alpha$, we denote (cf. [8, p. 157])

$$
\begin{aligned}
& \left(\mathbb{D}_{-, \varepsilon}^{\alpha} \psi\right)(u)=\frac{1}{\varkappa_{\ell}(\alpha)} \int_{\varepsilon}^{\infty}\left[\sum_{j=0}^{\ell}\left(\begin{array}{l}
\ell \\
j
\end{array}\right)(-1)^{j} \psi(u+j t)\right] \frac{d t}{t^{1+\alpha}}, \quad \varepsilon>0, \\
& \varkappa_{\ell}(\alpha)=\int_{0}^{\infty}\left(1-e^{-t}\right)^{\ell} t^{-1-\alpha} d t \\
& =\left\{\begin{array}{l}
\Gamma(-\alpha) \sum_{j=1}^{\ell}\left(\begin{array}{l}
\ell \\
j
\end{array}\right)(-1)^{j} j^{\alpha}, \quad \alpha \neq 1,2, \ldots, \ell-1, \\
\frac{(-1)^{1+\alpha}}{\alpha !} \sum_{j=1}^{\ell}\left(\begin{array}{l}
\ell \\
j
\end{array}\right)(-1)^{j} j^{\alpha} \log j, \quad \alpha=1,2, \ldots, \ell-1 .
\end{array}\right.
\end{aligned}
$$

The (right-sided) Marchaud fractional derivative of $\psi$ of order $\alpha$ is defined by $\mathbb{D}_{-}^{\alpha} \psi=\lim _{\varepsilon \rightarrow 0} \mathbb{D}_{-, \varepsilon}^{\alpha} \psi$. Due to normalization, this limit is independent of the choice of $\ell>\alpha$ (see, e.g., [8] or the proof of Theorem 2.2 below). The fact that $\mathbb{D}_{-}^{\alpha} \psi$ is independent of $\ell>\alpha$ becomes transparent if we set $\psi(u)=e^{-u}$, so that $\left(\mathbb{D}_{-}^{\alpha} \psi\right)(u)=e^{-u}$ (in the general case this fact can be proved, e.g., by applying the Fourier transform).

Theorem 2.2. Let $\varphi=\hat{f}, f \in L^{p}\left(\mathbb{H}^{n}\right),(k-1) /(n-1)<1 / p \leq 1$. Then

$$
\begin{gathered}
f(x)=\frac{\pi^{-k / 2}}{\varkappa_{\ell}(k / 2)} \int_{0}^{\infty}\left[\sum_{j=0}^{\ell}\left(\begin{array}{l}
\ell \\
j
\end{array}\right)(-1)^{j}(1+j t)^{(k-1) / 2} \check{\varphi}_{\cosh ^{-1} \sqrt{1+j t}}(x)\right] \frac{d t}{t^{1+k / 2}}, \\
\int_{0}^{\infty}=\lim _{\varepsilon \rightarrow 0} \int_{\varepsilon}^{\infty},
\end{gathered}
$$

where the limit is understood in the $L^{p}$-norm and in the a.e. sense. If $f \in C_{0} \cap L^{p}$, then $\lim _{\varepsilon \rightarrow 0}$ can be treated in the sup-norm.

Remark 2.3. The formula (2.12) is a regularized version of Helgason's formula (1.2) for $f \in L^{p}$. The right-hand side of (2.12) exactly represents Marchaud's fractional derivative of $\psi_{x}(u)$ (see (2.6)) evaluated for $u=1$.

Proof. For $\alpha=k / 2$ we have

$$
\sum_{j=0}^{\ell}\left(\begin{array}{l}
\ell \\
j
\end{array}\right)(-1)^{j}\left(I_{-}^{\alpha} g_{x}\right)(1+j t)=t^{\alpha} \int_{0}^{\infty} k(u) g_{x}(1+u t) d u
$$

$k(u)=\frac{1}{\Gamma(\alpha)} \sum_{j=0}^{\ell}\left(\begin{array}{l}\ell \\ j\end{array}\right)(-1)^{j}(u-j)_{+}^{\alpha-1}$, and therefore

$$
\left(\mathbb{D}_{-, \varepsilon}^{\alpha} \psi_{x}\right)(1)=\left(\mathbb{D}_{-, \varepsilon}^{\alpha} I_{-}^{\alpha} g_{x}\right)(1)=\int_{0}^{\infty} \lambda_{\ell, \alpha}(\eta) g_{x}(1+\varepsilon \eta) d \eta,
$$


where

$$
\lambda_{\ell, \alpha}(\eta)=\left[\varkappa_{\ell}(\alpha) \Gamma(1+\alpha) \eta\right]^{-1} \sum_{j=0}^{\ell}\left(\begin{array}{l}
\ell \\
j
\end{array}\right)(-1)^{j}(\eta-j)_{+}^{\alpha} .
$$

It is known [8, Lemma 10.17] that

$$
\int_{0}^{\infty} \lambda_{\ell, \alpha}(\eta) d \eta=1, \quad \lambda_{\ell, \alpha}(\eta)=\left\{\begin{array}{l}
O\left(\eta^{\alpha-1}\right), \quad \eta<1, \\
O\left(\eta^{\alpha-\ell-1}\right), \quad \eta>1 .
\end{array}\right.
$$

Owing to $(2.7),(2.13)$ yields

$\left(\mathbb{D}_{-, \varepsilon}^{\alpha} \psi_{x}\right)(1)=\frac{2}{\sigma_{n-1}} \int_{X} f(y) k_{\varepsilon}([x, y]) d y, \quad k_{\varepsilon}(\tau)=\frac{\left(\tau^{2}-1\right)^{1-n / 2}}{\varepsilon} \lambda_{\ell, \alpha}\left(\frac{\tau^{2}-1}{\varepsilon}\right)$,

and application of Theorem 2.3 (on approximate identity) from [2] gives the required result.

Theorem A is a particular case of Theorem 2.2 for $k=1$.

2.2. The case $X=S^{n}$. In this case an analog of (2.6) reads

$$
\begin{gathered}
\left(I_{0+}^{k / 2} g_{x}\right)(u)=\psi_{x}(u), \\
g_{x}(v)=v^{-1 / 2}\left(M_{\sqrt{v}} f\right)(x), \quad \psi_{x}(u)=\frac{u^{(k-1) / 2}}{2 \pi^{k / 2}} \check{\varphi}_{\cos ^{-1} \sqrt{u}}(x),
\end{gathered}
$$

with the left-sided (!) fractional integral $\left(I_{0+}^{\alpha} g\right)(u)=\frac{1}{\Gamma(\alpha)} \int_{0}^{u} g(v)(u-v)^{\alpha-1} d v$ and

$$
\left(M_{t} f\right)(x)=\frac{\left(1-t^{2}\right)^{(1-n) / 2}}{\sigma_{n-1}} \int_{\left\{y \in S^{n}: x \cdot y=t\right\}} f(y) d \sigma(y),
$$

$x \cdot y=x_{1} y_{1}+\ldots+x_{n+1} y_{n+1}$. Now we proceed as in Section 2.1 with the following changes: we extend both sides of (2.14) to $u<0$ by zero, and then apply the "left-sided" Marchaud's fractional derivative

$$
\left(\mathbb{D}_{+}^{\alpha} \psi\right)(u)=\lim _{\varepsilon \rightarrow 0} \frac{1}{\varkappa_{\ell}(\alpha)} \int_{\varepsilon}^{\infty}\left[\sum_{j=0}^{\ell}\left(\begin{array}{l}
\ell \\
j
\end{array}\right)(-1)^{j} \psi(u-j t)\right] \frac{d t}{t^{1+\alpha}} .
$$

By making use of approximation to the identity (see [11, Lemma 2.2] or [9, Lemma 2.9]) we get the following analog of Theorem 2.2.

Theorem 2.4. Let $\varphi=\hat{f}, f \in L^{p}\left(S^{n}\right), 1 \leq p<\infty$. Then

$$
\begin{gathered}
f(x)=\frac{\pi^{-k / 2}}{2 \varkappa_{\ell}(k / 2)} \int_{0}^{\infty}\left[\sum_{j=0}^{\ell}\left(\begin{array}{l}
\ell \\
j
\end{array}\right)(-1)^{j}(1-j t)_{+}^{(k-1) / 2} \check{\varphi}_{\cos ^{-1} \sqrt{1-j t}}(x)\right] \frac{d t}{t^{1+k / 2}}, \\
\int_{0}^{\infty}=\lim _{\varepsilon \rightarrow 0} \int_{\varepsilon}^{\infty},
\end{gathered}
$$

where the limit is understood in the $L^{p}$-norm and in the a.e. sense. If $f \in C\left(S^{n}\right)$, then $\lim _{\varepsilon \rightarrow 0}$ can be treated in the sup-norm.

Theorem B is a particular case of Theorem 2.4 for $k=1$. 


\section{INVERSION FORMULAE WITH DERIVATIVES}

We restrict ourselves by the case $X=\mathbb{H}^{n}$ (the case $X=S^{n}$, which was studied in [9], is simpler). Fractional integrals $\psi_{x}(u)=\left(I_{-}^{k / 2} g_{x}\right)(u)$ in $(2.6)$ are $L^{q}$-valued functions of $u$ for some $q$, and their derivatives

$$
\frac{d}{d u} \psi_{x}(u)=\lim _{\delta \rightarrow 0} \frac{\psi_{x}(u+\delta)-\psi_{x}(u)}{\delta}
$$

can be defined in a natural way by setting $\lim =\stackrel{L^{q}}{\lim }$ or $\lim =\lim$ (in the $x$-variable). In accordance with this definition we have the following:

Lemma 3.1. Let $g_{x}$ be defined by (2.7) with $f \in L^{p}$. Then we have the following:

(i) If $1 \leq p<n-1$, then $\left.\left(-\frac{d}{d u}\right)\left(I_{-}^{1} g_{x}\right)(u)\right|_{u=1}=f(x)$, the derivative being interpreted in the $L^{p}$-norm or in the a.e. sense.

(ii) If $0 \leq 1 / q<1 / p-(2 \alpha-3) /(n-1)$, then for each $u \geq 1,\left(-\frac{d}{d u}\right)\left(I_{-}^{\alpha} g_{x}\right)(u)=$ $\left(I_{-}^{\alpha-1} g_{x}\right)(u)$ in the $L^{q}$-norm or in the a.e. sense.

Proof. (i) For $\psi=I_{-}^{1} g_{x}$ the required derivative can be expressed through approximate identity. Namely,

$$
\begin{gathered}
\lim _{\delta \rightarrow 0} \frac{\psi(1)-\psi(1+\delta)}{\delta}=\lim _{\delta \rightarrow 0} \frac{1}{\delta} \int_{1}^{1+\delta} M_{\tau} f \frac{d \tau}{\sqrt{\tau}}=\frac{2}{\sigma_{n-1}} \lim _{\delta \rightarrow 0} \int_{X} k_{\delta}([x, y]) f(y) d y, \\
k_{\delta}(\tau)=\frac{\left(\tau^{2}-1\right)^{1-n / 2}}{\delta} \chi\left(\frac{\tau^{2}-1}{\delta}\right), \quad \chi(s)=\left\{\begin{array}{lll}
1 & \text { if } & 0<s<1, \\
0 & \text { if } & s>1,
\end{array}\right.
\end{gathered}
$$

and the result follows by Theorem 2.3 from [2].

(ii) We have to show that the expression

$$
\frac{1}{\delta}\left[\left(I_{-}^{\alpha} g_{x}\right)(u)-\left(I_{-}^{\alpha} g_{x}\right)(u+\delta)\right]-\left(I_{-}^{\alpha-1} g_{x}\right)(u)
$$

tends to 0 as $\delta \rightarrow 0$ in the required sense. It can be written as a convolution $\frac{2}{\sigma_{n-1}}\left(f * h_{\delta}\right)(x)$ where

$$
\begin{aligned}
h_{\delta}(\tau) & =\left(\tau^{2}-1\right)^{1-n / 2}\left[\frac{\left(\tau^{2}-u\right)_{+}^{\alpha-1}-\left(\tau^{2}-u-\delta\right)_{+}^{\alpha-1}}{\Gamma(\alpha) \delta}-\frac{\left(\tau^{2}-u\right)_{+}^{\alpha-2}}{\Gamma(\alpha-1)}\right] \\
& =\frac{\left(\tau^{2}-1\right)^{1-n / 2}\left(\tau^{2}-u\right)_{+}^{\alpha-2}}{\Gamma(\alpha-1)} k\left(\frac{\delta}{\tau^{2}-u}\right), \quad k(\omega)=\frac{1-(1-\omega)_{+}^{\alpha-1}}{(\alpha-1) \omega}-1 .
\end{aligned}
$$

The function $k(\omega)$ is bounded for $\omega>0$ and $\lim _{\omega \rightarrow 0} k(\omega)=0$. Hence by Young's inequality, $\left\|f * h_{\delta}\right\|_{q} \leq\|f\|\left\|_{p}\right\| h_{\delta} \|_{r}$ with the required $q$ (use (2.9) with $\alpha$ replaced by $\alpha-1)$. Now the Lebesgue theorem on dominated convergence yields

$$
\lim _{\delta \rightarrow 0}\left\|h_{\delta}\right\|_{r}^{r}=0, \quad \lim _{\delta \rightarrow 0}^{\text {a.e. }}\left(f * h_{\delta}\right)(x)=0
$$

and we are done.

Lemma 3.1 leads to the following

Definition 3.2. We set

$$
\mathcal{D}_{m}=(-1)^{m} \partial_{m} \partial_{m-1} \ldots \partial_{1},
$$


where all derivatives $\partial_{i}$ designate the same expression $\frac{d}{d u}$, but their interpretation depends on $i$. Namely, if $i<k / 2$, then $\partial_{i}$ is understood in the $L^{q_{i}}$-norm where

$$
0 \leq \frac{1}{q_{i}}<\frac{1}{p}-\frac{k-1}{n-1}+\frac{2 i}{n-1} .
$$

If $i=k / 2$, then $\partial_{i}$ is understood in the $L^{p}$-norm. Alternatively all derivatives in (3.1) may be treated in the a.e. sense.

Theorem 3.3. Let $\varphi=\hat{f}, f \in L^{p},(k-1) /(n-1)<1 / p \leq 1$,

$$
\tilde{\psi}_{m}(x, u)=\pi^{-k / 2} \mathcal{D}_{m}\left[u^{(k-1) / 2} \check{\varphi}_{\cosh ^{-1} \sqrt{u}}(x)\right], \quad m \in \mathbb{N} .
$$

Then we have the following:

(i) If $k=2 m$, then $f(x)=\tilde{\psi}_{m}(x, 1)$.

(ii) If $k=2 m+1$, then

$$
f(x)=\frac{1}{2 \pi^{1 / 2}} \int_{0}^{\infty}\left[\tilde{\psi}_{m}(x, 1)-\tilde{\psi}_{m}(x, 1+t)\right] \frac{d t}{t^{3 / 2}}, \quad \int_{0}^{\infty}=\lim _{\varepsilon \rightarrow 0} \int_{\varepsilon}^{\infty},
$$

where the limit is understood as in Theorem 2.2.

Proof. (i) follows immediately from Lemma 3.1 and Definition 3.2. In order to prove (ii), by Lemma 3.1 we have $\left(I_{-}^{1 / 2} g_{x}\right)(u)=\tilde{\psi}_{m}(x, u)$. As in the proof of Theorem 2.2 , application of Marchaud's derivative $\mathbb{D}_{-}^{1 / 2}$ yields

$$
f(x)=\frac{1}{\varkappa_{1}(1 / 2)} \int_{0}^{\infty}\left[\tilde{\psi}_{m}(x, 1)-\tilde{\psi}_{m}(x, 1+t)\right] \frac{d t}{t^{3 / 2}}, \quad \varkappa_{1}(1 / 2)=2 \pi^{1 / 2} .
$$

This completes our investigation.

\section{REFERENCES}

[1] C.A. Berenstein, E. Casadio Tarabusi, Inversion formulas for the $k$-dimensional Radon transform in real hyperbolic spaces, Duke Math. Journal 62 (1991), 613-631. MR 93b:53056

[2] C.A. Berenstein, B. Rubin, Radon transform of $L^{p}$-functions on the Lobachevsky space and hyperbolic wavelet transforms, Forum Math. 11 (1999), 567-590. MR 2000e:44001

[3] Totally geodesic Radon transform of $L^{p}$-functions on real hyperbolic space, Preprint, 2001.

[4] S. Helgason, Differential operators on homogeneous spaces, Acta Math. 102 (1959), 239-299. MR 22:8457

[5] The totally geodesic Radon transform on constant curvature spaces, Contemp. Math 113 (1990), 141-149. MR 92j:53036

[6] The Radon transform, Birkhäuser, Boston, Second edition, 1999. MR 2000m:44003

[7] D. M. Oberlin, E. M. Stein, Mapping properties of the Radon transform, Indiana Univ. Math. J. 31 (1982), 641-650. MR 84a:44002

[8] B. Rubin, Fractional integrals and potentials, Pitman Monographs and Surveys in Pure and Applied Mathematics, 82, Longman, Harlow, 1996. MR 98h:42018

[9] Inversion formulas for the spherical Radon transform and the generalized cosine transform, Advances in Appl. Math. (to appear).

[10] _ Radon, cosine, and sine transforms on real hyperbolic space, Advances in Math. (to appear).

[11] _ Spherical Radon transforms and related wavelet transforms, Applied and Computational Harm. Anal. 5 (1998), 202-215. MR 99c:44003

[12] R.S. Strichartz, $L^{p}$-estimates for Radon transforms in Euclidean and non-euclidean spaces, Duke Math. J. 48 (1981), 699-727. MR 86k:43008

Institute of Mathematics, Hebrew University, Jerusalem 91904, Israel

E-mail address: boris@math.huji.ac.il 